\title{
Diabetic macular edema and its association to systemic risk factors in an urban north Indian population.
}

\author{
Singh Luxmi*, Mullick Ritika, Ahmed Lubna, Garg Pragati, Lal B.B \\ Department of Ophthalmology, Era’s Lucknow Medical College and Hospital, India
}

\begin{abstract}
Aim: To study the association of systemic risk factors with diabetic macular edema in an urban North Indian population.

Materials and Methods: 600 eyes of 300 patients in the age group of 30-70 years with pre-diagnosed diabetes mellitus type II were screened for diabetic retinopathy and diabetic macular edema. All systemic parameters were studied.

Results: Diabetic retinopathy was found in $52 \%$ males with the male to female ratio being 1.08:1. Cystoid macular edema and serous retinal detachment were associated with increased fasting and post prandial blood sugar levels respectively. Hypertriglyceridemia was significantly associated with cystoid macular edema ( $p$-value $=\mathbf{0 . 0 0 1}$ ). $73.7 \%$ of smokers had cystoid macular edema. Increased serum creatinine is more commonly found in cystoid macular edema. Higher levels of Blood urea is significantly associated with diffuse retinal thickening ( $p$-value $=0.006$ )

Conclusion: Of the systemic risk factors found in the diabetic patients, macular edema was found more in females, smokers, those with uncontrolled diabetes mellitus, deranged lipid profile and nephropathy.
\end{abstract}

Keywords: Diabetic macular edema, Diabetic retinopathy, Hyporeflective cavity

Accepted on October 03, 2018

\section{Introduction}

Diabetic macular edema occurs due to the retinal micro vascular changes which in turn compromise the blood retinal barrier, causing leakage of plasma constituents into the surrounding retinal tissue leading to retinal edema. There is also loss of pericytes and endothelial cell junction dysfunction which leads to extravasation of fluid and results in edema. Diabetic macular edema was earlier defined as retinal thickening within 2-disc diameters of the centre of the macula [1].

Currently, the ETDRS defines clinically significant macular edema as the presence of: 1) thickening of the retina within 500 $\mu \mathrm{m}$ of the centre of the fovea, 2) hard exudates within $500 \mu \mathrm{m}$ of the centre of the fovea. 3) an area of 1 disc of diameter of retinal thickening that intercepts the area defined by one disc diameter of radius centred at the fovea.

Its prevalence has been reported to follow a varying range in different studies. It affects central vision from the early stages of retinopathy, and it is the most frequent sight-threatening complication of diabetic retinopathy, particularly in older type 2 diabetic patients. The role of diabetic macular edema in vision loss of diabetic patients and its occurrence in the evolution of the retinal disease are being increasingly recognized [2]. Diabetic Macular Edema (DME) is one of the leading causes of blindness in the diabetic population.

Optical Coherence Tomography enables precise measurement of macular thickness and facilitates detecting macular oedema which is the main pathologic feature of diabetic maculopathy.
Early Treatment of Diabetic Retinopathy Study (ETDRS) have categorised the severity of macular edema and the term Clinically Significant Macular Edema (CSME) has been defined for treatment guidelines. CSME is defined as any detectable retinal thickening due to fluid accumulation (ETDRS report). The edema over the macula may start as a focal lesion, which later progresses to a more diffuse state. Cystoid cavities are formed due to persistent retinal edema which may result in Muller cell necrosis. These cystoid cavities are located in the outer layer of retina (Henle's fibre and outer plexiform layer), and sometimes also in the inner plexiform layer.

Several central cysts merging together forming large hyporeflective cavity which contribute to significant thickening of the fovea is commonly seen in well-established long standing cases of macular edema. The main characteristics of macular oedema in OCT, apart from increased retinal thickness, disintegration of the layered retinal structure, include intraretinal spaces of reduced reflectivity and usually also flattening of the central foveal depression. In some cases fluid can be seen under the neurosensory retina. OCT tomograms can also reveal hard exudates and haemorrhages.

Diabetic macular edema may be classified based on Optical Coherence Tomography (OCT) measurements. Using OCT, DME can be classified into four main types [3] presented as follows:

Type 1: Early diabetic macular edema.

Type 2: Simple diabetic macular edema. 
Type 3: Cystoid diabetic macular edema, further divided into 3a, mild; 3b, intermediate; $3 c$, severe.

Type 4: Serous macular detachment.

The known risk factors for diabetic macular edema are male sex, more severe diabetic retinopathy, higher glycosylated haemoglobin, proteinuria, higher systolic and diastolic blood pressure, more pack-years of smoking, longer duration of diabetes and elevated serum globulin level.

In planning the needs and entering the cases associated with treatment modalities, it is important to ascertain an accurate estimate of the magnitude of and risk factors for diabetic macular edema in a setting, so with this in mind, the present study was undertaken.

In the backdrop of these associations of systemic risk factors with diabetic macular edema, we decided to study this in further detail.

\section{Materials and Methods}

This cross-sectional observational study was done over the duration of 18 months in a tertiary care hospital of North India, where 600 eyes of 300 patients were observed following the exclusion criteria. The study population included prediagnosed cases of diabetes mellitus type II with diabetic retinopathy between the age group of 30-70 years. The exclusion criteria for the study was patients suffering from any other macular pathology, opaque or hazy ocular media which would prevent fundus visualisation.

A detailed history including age of the patient, duration \&course of diabetes, smoking and if the patient is on oral hypoglycaemics or insulin was obtained. General physical examination was performed, which included the measurement of the blood pressure, body mass index calculation, followed by biochemical evaluation of blood sugar (fasting and PP), $\mathrm{HbA1c}$, lipid profile, renal function tests (blood urea and serum creatinine) and urine examination (sugar and albumin).

The biochemical evaluation was done by obtaining $2 \mathrm{~mL}$ of blood sample from the patient in a sterile vial and sent to the Department of Biochemistry. All the biochemical assessments were done using an Autoanalyzer. Thorough ocular evaluation was done on all selected patients both clinically as well as with the help of diagnostic instruments. Both Uncorrected and best corrected visual acuity was recorded using a Snellen's chart. Anterior segment evaluation was done using diffuse light and slit lamp examination to look for any other ocular disease or ocular surgery. Fundus examination was performed by Direct Ophthalmoscopy, Indirect Ophthalmoscopy and +90D lenses. The intraocular pressure was measured using an applanation tonometer. Optical coherence tomography was performed using Cirrus 500 machine manufactured by Carl-Zeiss,
Germany to measure the macular thickness. Using the retinal thickness map analysis protocol, macular thickness was determined and compared with normative data. Signs of macular edema were noted for confirmation of diagnosis. It consists of a macular cube $512 \times 128$ (vertical $\times$ horizontal) axial scans covering an area of $6 \times 6 \mathrm{~mm}$ in the macular region. The macula was divided into 3 concentric circles centered at the fovea. This division is a superimposition of the ETDRS map over the OCT map of the macula. It consists of 3 zones; the fovea (less than $1 \mathrm{~mm}$ diameter), the inner macula (1 to 3 $\mathrm{mm}$ ) and the outer macula (3 to $6 \mathrm{~mm}$ ).

The inner and the outer ring are further divided into four equal regions. The innermost $1 \mathrm{~mm}$ ring is the fovea while the $3 \mathrm{~mm}$ inner ring and $6 \mathrm{~mm}$ outer ring are further divided into four equal regions, including the superior, inferior, nasal and temporal quadrant. It identifies the layers of the retina and determines macular thickness by measuring the distance between the Inner Limiting Membrane (ILM) and the inner boundary of Retinal Pigment Epithelium (RPE).

The data was analysed using Statistical Package for Social Sciences, version 15.0. For, categorical data Chi-square test was used whereas continuous data was analysed using ANOVA and student T-test. Multivariate assessment was done using logistic regression. The confidence level of the study was kept at $95 \%$ and hence a P-value less than 0.05 indicated a statistically significant association.

The research followed the tenets of Declaration of Helsinki; IRB approval and informed consents were obtained after explanation of the study. The institutional review board of the College approved the study.

\section{Results}

This cross-sectional study was carried out on 300 (600 eyes) prediagnosed cases of diabetes with diabetic retinopathy in age group of 30-70 year were enrolled. 300 patients who were prediagnosed diabetics were enrolled in this study. The patients were from the age of 30 to 70 years. The cases having any confounding factors such as macular pathology, opaque/hazy ocular media preventing fundus visualization and co-existing ocular disorders likely to mask the findings of diabetic macular edema were not included in the assessment. Thorough demographic, general, biochemical and ocular evaluation was done in all the cases. History and clinical status of disease was noted. Majority of patients enrolled in the study were males $(52 \%)$. The male to female ratio of the study patient's was 1.08:1. A total of 87 eyes had diabetic macular edema. Out of these, majority $(59.8 \%)$ had diffuse retinal thickening. Cystoid macular edema was seen in $21.8 \%$ and serous retinal detachment in $18.4 \%$ (Table 1 ).

Table 1. Distribution of patients according to Patterns of DME (EDTR) $(n=87)$

\begin{tabular}{|l|l|l|l|}
\hline S.No. & Characteristic & No. of Patients & Percentage \\
\hline 1. & Diffuse retinal thickening & 52 & 59.8 \\
\hline
\end{tabular}


Citation: Luxmi. S, Ritika. M, Lubna. A, et al.. Diabetic macular edema and its association to systemic risk factors in an urban north Indian population. J Clin Ophthalmol. 2018;2(2):86-91.

\begin{tabular}{|l|l|l|l|}
\hline 2. & Cystoid macular edema & 19 & 21.8 \\
\hline 3. & Serous retinal detachment & 16 & 18.4 \\
\hline
\end{tabular}

$42.3 \%$ of males and $30 \%$ of females showed diffuse retinal thickening. $100 \%$ of females showed cystoid macular edema, while no males in the study showed this type of macular edema. $43.8 \%$ of males and $56.3 \%$ of females showed serous retinal detachment.

On looking for association between the patterns of diabetic macular edema with various systemic risk factors it was found that cystoid macular edema was significantly associated with females and patients with hypertriglyceridemia.

Cystoid macular edema and serous retinal detachment was associated with increased levels of blood sugar of fasting and post prandial respectively. $63.2 \%$ of patients on Insulin treatment showed cystoid macular edema. Of those who were smokers, $73.7 \%$ had cystoid macular edema. Increase in serum creatinine is more commonly found in cystoid macular edema. Serous retinal detachment was significantly associated with intake of oral hyperglycemic agents (Figure 1).

\section{Distribution of patients according to Patterns of DME (EDTR) $(n=87)$}

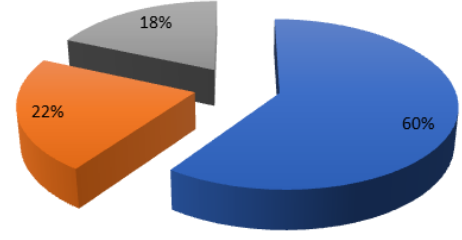

- Diffuse retinal thickenin - Cystoid macular edema - Serous retinal detachment

Figure 1.Distribution of patients according to Patterns of DME (EDTR) $(n=87)$

Out of 300, 160 patients were overweight or obese, majority of the patients showed diffuse retinal thickening. Higher levels of Blood urea are significantly associated with diffuse retinal thickening ( $\mathrm{p}$-value $=0.006)$

The present study was bound by a sampling frame where only those patients aged 30-70 years were included and within this sampling frame, we found that in the age group of 40-50 years, $47.4 \%$ were in the group of cystoid macular edema. Among patients of $50-60$ years, $57.7 \%$ showed diffuse retinal thickening and $56.3 \%$ had serous retinal detachment $(p=0.569)$.

Majority of patients had diabetes for past more than 10 years $(70.7 \%)$. In cases where the disease was between $10-20$ years, $63.5 \%$ showed diffuse retinal thickening. 21-40 years of disease had $47.4 \%$ of cystoid macular edema and $31.3 \%$ of serous retinal detachment $(\mathrm{p}=0.367)$.

A total of $145(48.3 \%)$ patients were on Oral Hypoglycemic Agents (OHAs), 120 (40\%) were on insulin. Amongst the cases on oral hyperglycemic agents $75 \%$ showed serous retinal detachment. The cases on insulin, $63.2 \%$ had a positive correlation with cystoid macular edema $(\mathrm{p}=0.003)$.

Blood sugar fasting levels of $>125 \mathrm{mg} / \mathrm{dl}$, the diffuse retinal thickening was $59.6 \%$, cystoid macular edema was $89.5 \%$ and serous retinal detachment was seen to be $81.3 \%(\mathrm{p}=0.029)$. The blood sugar post prandial levels of $>200 \mathrm{mg} / \mathrm{dl}, 50 \%$ showed diffuse retinal thickening, $42.1 \%$ showed cystoid macular edema and $100 \%$ showed serous retinal detachment $(p=0.001)$.

The proportion of those with uncontrolled diabetes was relatively much higher with $84.3 \%$ patients having $\mathrm{HbA} 1 \mathrm{c}$ levels $>8.5 \%(p=0.441)$. Similar to results of present study, Raman et al. [4] also observed that those taking OHAs comprised the maximum proportion of their DR population. In another study, Raman et al. [4] reported that higher $\mathrm{HbA1c}$ levels are associated with a higher risk of DR. In this study HbAlc levels $>8.5 \%$, 52.6\% had cystoid macular edema. While levels between $7.1 \%$ to $8.5 \%$ had positive correlation to diffuse retinal thickening $46.2 \%$ and serous retinal detachment $56.3 \%(\mathrm{p}=0.441)$.

In present study, $26 \%$ patients were smokers and $15 \%$ patients had a positive family history. Of these patients, $67.3 \%$ had diffuse retinal thickening, $73.7 \%$ had cystoid macular edema and $25 \%$ had serous retinal detachment $(\mathrm{p}=0.004)$.

More than half $(53.3 \%)$ patients were overweight or obese , $55.8 \%$ of overweight patients had positive correlation with diffuse retinal thickening, $42.1 \%$ had cystoid macular edema and $50 \%$ had serous retinal detachment $(\mathrm{p}=0.022)$.

Among lipid abnormalities, triglyceridemia was most common, seen in $65 \%$ patients and showed a positive correlation of $63.2 \%$ to cystoid macular edema $(p=0.001)$. All these factors are commonly associated with lifestyle disorders and are referred to as the metabolic syndrome factors. The association between metabolic syndrome and diabetic retinopathy has been shown in several case series [5-7] and findings of the present study reendorse them.

In present study, blood urea was above normal level in $15 \%$, where $57.7 \%$ patients in this study showed a positive correlation to diffuse retinal thickening $(\mathrm{p}=0.006)$ (Table 2$)$.

Table 2. Association between various patterns of Macular Edema with risk factors in diabetic retinopathy $(n=87)$.

\begin{tabular}{|l|l|l|l|l|}
\hline S.No & Risk Factors & $\begin{array}{l}\text { Diffuse retinal } \\
\text { thickening }(n=52)\end{array}$ & $\begin{array}{l}\text { Cystoid macular edema } \\
(n=19)\end{array}$ & $\begin{array}{l}\text { Serous } \\
\text { detachment }(n=16)\end{array}$ \\
\hline
\end{tabular}




\begin{tabular}{|c|c|c|c|c|c|c|c|c|c|}
\hline & & No. & $\%$ & No. & $\%$ & No. & $\%$ & $x^{2}$ & 'p' \\
\hline \multirow[t]{3}{*}{1} & Gender & & & & & & & & \\
\hline & Male & 22 & 42.3 & 0 & 0 & 7 & 43.8 & 12.166 & $<0.001$ \\
\hline & Female & 30 & 57.7 & 19 & 100 & 9 & 56.3 & & \\
\hline \multirow[t]{4}{*}{2} & Treatment & & & & & & & & \\
\hline & $\mathrm{OHA}$ & 22 & 42.3 & 4 & 21.1 & 12 & 75 & 16.423 & 0.003 \\
\hline & Insulin & 24 & 46.2 & 12 & 63.2 & 0 & 0 & & \\
\hline & Not on treatment & 6 & 11.5 & 3 & 15.8 & 4 & 25 & & \\
\hline \multirow[t]{3}{*}{3} & Blood sugar Fasting levels & & & & & & & & \\
\hline & $100-125 \mathrm{mg} / \mathrm{dl}$ & 21 & 40.4 & 2 & 10.5 & 3 & 18.8 & 7.081 & 0.029 \\
\hline & $>125 \mathrm{mg} / \mathrm{dl}$ & 31 & 59.6 & 17 & 89.5 & 13 & 81.3 & & \\
\hline \multirow[t]{3}{*}{4} & Blood sugar PP levels & & & & & & & & \\
\hline & $<200 \mathrm{mg} / \mathrm{dl}$ & 26 & 50 & 11 & 57.9 & 0 & 0 & 14.86 & 0.001 \\
\hline & $>200$ mg/dl & 26 & 50 & 8 & 42.1 & 16 & 100 & & \\
\hline 5 & Smoking & 35 & 67.3 & 14 & 73.7 & 4 & 25 & 10.86 & 0.004 \\
\hline \multirow[t]{5}{*}{6} & BMI category & & & & & & & & \\
\hline & Underweight $\left(<18.5 \mathrm{~kg} / \mathrm{m}^{2}\right)$ & - & - & - & - & - & - & 11.415 & 0.022 \\
\hline & Normal $\left(18.5-25.0 \mathrm{~kg} / \mathrm{m}^{2}\right)$ & 7 & 13.5 & 9 & 47.4 & 6 & 37.5 & & \\
\hline & Overweight $\left(25.0-30 \mathrm{~kg} / \mathrm{m}^{2}\right)$ & 29 & 55.8 & 8 & 42.1 & 8 & 50 & & \\
\hline & Obese $\left(>30 \mathrm{~kg} / \mathrm{m}^{2}\right)$ & 16 & 30.8 & 2 & 10.5 & 2 & 12.5 & & \\
\hline \multirow[t]{5}{*}{7} & Triglycerides (mg/dl) & & & & & & & & \\
\hline & $<150$ & 24 & 46.2 & 3 & 15.8 & 6 & 37.5 & 19.713 & 0.001 \\
\hline & $150-199$ & 27 & 51.9 & 12 & 63.2 & 4 & 25 & & \\
\hline & 200- 499 & 1 & 1.9 & 4 & 21.1 & 6 & 37.5 & & \\
\hline & $>500$ & & & & & & & & \\
\hline \multirow[t]{3}{*}{8} & Blood urea (mg/dl) & & & & & & & & \\
\hline & $20-40$ & 22 & 42.3 & 16 & 84.2 & 10 & 62.5 & 10.305 & 0.006 \\
\hline & $>40$ & 30 & 57.7 & 3 & 15.8 & 6 & 37.5 & & \\
\hline
\end{tabular}

Serum creatinine levels of $<0.8 \mathrm{mg} / \mathrm{dl}, 98.1 \%$ had diffuse retinal thickening, $87.5 \%$ had serous retinal detachment and $78.9 \%$ had cystoid macular edema $(\mathrm{p}=0.025)$.

The present study was limited to study of diabetic macular edema and its patterns in a highly specific risk group of diabetic retinopathy patients. It would be interesting to understand the patterns of DME in relation to different risk factors in diabetic patients without retinopathy or even in nondiabetic patients. Further studies on this aspect are warranted in order to highlight the relevance and importance of lifestyle factors in a non-diabetic or non-diabetic retinopathy population too.

\section{Discussion}

Diabetic Macular Edema (DME) is one of the leading causes of blindness and is a known progressive complication among patients with diabetic retinopathy Owing to severe vision threatening consequences associated with diabetic macular edema, it is a major cause of concern.

In the present study, macular edema was seen in 87 eyes $(14.5 \%)$. While in the study by Yau et al. [8] the prevalence of diabetic macular edema among diabetic retinopathy patients was found to be much lower 7.48\%. However, Wong et al. [9] reported DME in nearly $16.3 \%$ of diabetic retinopathy patients. In a recent metanalysis, Lee et al. [10] found prevalence of DME to range from $1.4 \%$ to $33.3 \%$ in different studies. The prevalence of DME in different studies depends on a host of factors including type of diabetes, duration of follow up, place 
Citation: Luxmi. S, Ritika. M, Lubna. A, et al.. Diabetic macular edema and its association to systemic risk factors in an urban north Indian population. J Clin Ophthalmol. 2018;2(2):86-91.

of study and presence of comorbidities. Ding and Wong [11] reported that nearly $29 \%$ of US adults with diabetes have diabetic retinopathy and of these nearly $10 \%$ have diabetic macular edema.

The present study specifically targets the prevalence of macular edema in diabetics having retinopathy; most of the other studies have targeted on the prevalence of diabetic macular edema among diabetic patients in general and have considered diabetic macular edema as an extended complication in diabetic retinopathy patients.

Our study targets the prevalence of macular edema in diabetic patients with retinopathy. Various other studies done, observe diabetic macular edema as a complication of diabetic retinopathy.

On looking at the pattern of DME amongst DR patients; we observed that of all the DME patients, majority (59.8\%) had diffuse retinal thickening. Cystoid macular edema was seen in $19(21.8 \%)$ and serous retinal detachment in 16 eyes $(18.4 \%)$. Similar to results of present study, diffuse retinal thickening was found to be the major finding in other studies too. The prevalence of serous retinal detachment in present study was also in accordance with other series where it varied from $15 \%$ to $31 \%, 93-96$. However, prevalence of cystoid macular edema was slightly lower in present study as compared to the finding of Otani et al. [12] 94 who reported it to be prevalent in $47 \%$ of patients. One of the reason for these differences in pattern findings of present study to that of others could be attributed to the fact that in present study we assigned only one pattern to an eye whereas in some other studies 94 more than one patterns were identified and reported from a single eye. However, in present study we considered only the dominating pattern.

No significant association of age and gender was observed by us with occurrence of macular edema. However, duration of diabetes, Post Prandial (PP) blood sugar levels, increased HbA1c levels, smoking, family history and increased blood pressure levels were significantly associated with an increased risk of DME. These observations are in agreement with the findings reported in other epidemiologic and metanalytical studies $[13,14]$.

The correlation of various Optical Coherence Tomography (OCT) patterns of Diabetic Macular Edema (DME) was studied by Ghosh et al. Mean duration of diabetes was $8.7 \pm$ 4.2 years. Mean serum globulin level was significantly higher $(p=0.018)$ in serous compared to non-serous group. Prevalence of serous DME was significantly high in those with serum globulin level $>3.5 \mathrm{gm} / \mathrm{dl}$ (prevalence ratio=3.01, $\mathrm{p}=0.040$ ). Significant correlation of central macular thickness was observed with duration of diabetes $(p=0.002, r=0.440)$.Visual acuity (logMAR) was correlated significantly with $\mathrm{HbA} 1 \mathrm{C}$ $(p=0.031, r=0.305)$. Increased serum globulin, a positive phase reactant of inflammation, was found significant independent risk factor for development of serous DME. This study did not identify any modifiable systemic factor for any of the OCT patterns in DME. DME also showed a significant association with blood urea and increased BMI levels in our study.

\section{Conclusion}

Cystoid macular oedema is more common in females, patients with increased blood sugar and TG levels, patients on insulin $\&$ smokers. Serous retinal detachment is seen more frequently in patients with increased blood sugar levels \& on oral hypoglycemic agents. Diffuse retinal thickening is more commonly met in cases with increased BMI, Blood Urea.

\section{References}

1. Albert DM, Jakobiec FA. Principles and practice of ophthalmology. 2nd ed. philadelphia: WB Saunders Co; 2000.

2. Lobo C, Pires I, Cunha-Vaz J. Diabetic macular edema. In: R. Bernardes and J. Cunha-Vaz (eds.), Optical Coherence Tomography, Biological and Medical Physics, Biomedical Engineering. 2012. Springer-Verlag Berlin Heidelberg, pp. $1-21$.

3. Koleva-Georgieva DN, Sivkova NP. Types of diabetic macular edema assessed by optical coherence tomography. Folia Med. 2008;50:30-8.

4. Raman R, Verma A, Pal SS, et al. Influence of glycosylated hemoglobin on sight-threatening diabetic retinopathy: a population-based study. Diabetes Res Clin Pract. 2011;92:168-73.

5. Gao L, Xin Z, Yuan MX, et al. High prevalence of diabetic retinopathy in diabetic patients concomitant with metabolic syndrome. PLoS One. 2016;11:e0145293.

6. Kim TK, Won JY, Shin JA, et al. The association of metabolic syndrome with diabetic retinopathy: The korean national health and nutrition examination survey 20082012. Chen C-L, ed. PLoS ONE. 2016;11:e0157006.

7. Raman R, Gupta A, Pal SS, et al. Prevalence of Metabolic Syndrome and its influence on microvascular complications in the Indian population with Type 2 Diabetes Mellitus. Sankara nethralaya diabetic retinopathy epidemiology and molecular genetic study (SN-DREAMS, report 14). Diabetol Metab Syndr. 2010;2:67.

8. Yau JW, Rogers SL, Kawasaki R, et al. Global prevalence and major risk factors of diabetic retinopathy. Diabetes Care. 2012;35:556-64.

9. Wong TY, Cheung N, Tay WT, et al. Prevalence and risk factors for diabetic retinopathy: the Singapore Malay Eye Study. Ophthalmology. 2008;115:1869-75.

10. Lee R, Wong TY, Subanayagam C. Epidemiology of diabetic retinopathy, diabetic macular edema and related vision loss. Eye Vis (Lond). 2015;2:17.

11. Ding J, Wong TY. Current epidemiology of diabetic retinopathy and diabetic macular edema. Curr Diab Rep. 2012; 12(4): 346-354.

12. Otani T, Kishi S, Maruyama Y. Patterns of diabetic macular edema with optical coherence tomography. Am J Ophthalmol. 1999;127:688-93.

13. Zhang X, Zeng H, Bao S, et al. Diabetic macular edema: New concepts in patho-physiology and treatment. Cell Biosci. 2014;4:27. 
14. Ding J, Wong TY. Current epidemiology of diabetic retinopathy and diabetic macular edema. Curr Diab Rep. 2012;12:346-54.

\section{Correspondence to:}

Singh Luxmi
Department of Ophthalmology

Era's Lucknow Medical College and Hospital, Lucknow

E-mail: drluxmi@rediffmail.com

Tel: +91-9628727420 